\title{
Significance of pulmonary resection in patients with metachronous pulmonary metastasis from pancreatic ductal adenocarcinoma: a retrospective cohort study
}

Taro Mashiko*, Akira Nakano, Yoshihito Masuoka, Seiichiro Yamamoto, Soji Ozawa and Toshio Nakagohri

\begin{abstract}
Background: Pulmonary metastases from pancreatic ductal adenocarcinoma (PDAC) are relatively rare. Systemic chemotherapy is the first choice of treatment in patients with distant metastases, and the role of metastasectomy is controversial. The aim of the present study was to evaluate the outcome of patients with pulmonary metastases after resection of PDAC and the indications for metastasectomy.

Methods: We retrospectively analysed patients with pulmonary metastases as the first recurrence after resection of primary PDAC between January 2006 and December 2018. Clinical data were obtained from the patients' medical records. Relapse-free survival (RFS) and overall survival (OS) were analysed using the Kaplan-Meier method, and statistical significance was evaluated by the log-rank test.

Results: Of the 417 patients with resected PDACs, 24 (7.9\%) had pulmonary metastases. Six patients (25.0\%) underwent pulmonary resection and 18 (75.0\%) received systemic chemotherapy and best supportive care. There were no major complications requiring therapeutic intervention after pulmonary resection. The median RFS was 24.0 months (95\% Cl 10.8-37.2), and the 1-,3-, and 5-year RFS rates were $66.7 \%, 33.3 \%$, and $4.2 \%$, respectively. The median OS was 50.0 months (95\% Cl 15.9-84.1), and the 1-, 3-, and 5-year OS rates were 95.8\%, 70.3\%, and 46.4\%, respectively. All patients with resected pulmonary metastases were alive at the end of the study, whereas the median OS of the patients who did not undergo resection was 37.0 months (95\% Cl 34.4-39.6). Therefore, patients with resected pulmonary metastases had a significantly better prognosis $(p=0.008)$.

Conclusions: Pulmonary resection may improve the prognosis in selected patients with pulmonary metastases from PDAC. However, the present study is based on a small number of patients and may include a selection bias; therefore, a multi-institutional prospective study is needed to clarify the indications for pulmonary resection.
\end{abstract}

Keywords: Pancreatic ductal adenocarcinoma, Metachronous pulmonary metastasis, Pulmonary resection, Metastasectomy

*Correspondence: mt334592@tsc.u-tokai.ac.jp

Department of Gastroenterological Surgery, Tokai University School of Medicine, 143 Shimokasuya, Isehara, Kanagawa 259-1193, Japan

\section{Background}

Despite the development of multidisciplinary treatment for pancreatic ductal adenocarcinoma (PDAC), the prognosis for PDAC is still poor and the 5-year survival rate is less than $20 \%$ because of the high rate of recurrence [1, 2]. Even the most recent clinical trials of chemotherapy 
for distant metastases from PDAC have indicated a poor prognosis, with the median overall survival (OS) ranging from 8.5 months to 11.1 months [3, 4]. Recurrent PDAC often spreads to multiple organs. The most common site of PDAC metastasis is the liver, and the prognosis of livermetastatic PDAC is extremely poor [5]. Conversely, the incidence of metachronous pulmonary metastasis from PDAC is relatively low, at $2.9-21.8 \%$ [6-8]. Metachronous pulmonary metastases of PDAC are also reported to have a better prognosis than recurrent PDACs in other sites [9-11]. A few reports have suggested the feasibility and efficacy of surgical resection for isolated pulmonary metastases of PDAC [12-15]. However, the role of surgical resection of recurrent PDAC remains controversial. The aim of the present study was to evaluate the outcome of patients with pulmonary metastases after resection of PDAC and the indications for metastasectomy.

\section{Methods}

\section{Patients}

We retrospectively analysed patients with pulmonary metastases as the first recurrence after surgery for primary PDAC between January 2006 and December 2018 in the Tokai University Hospital, Japan. Staging of the primary PDAC was performed using the Union for International Cancer Control tumour-node-metastasis (TNM) classification, 8th edition. We excluded patients with synchronous distant metastases, those who had undergone conversion surgery and those with pancreatic pathologies other than PDAC, such as intraductal papillary mucinous carcinoma, adenosquamous carcinoma, carcinosarcoma, and anaplastic carcinoma.

\section{Follow-up}

All patients received routine postoperative surveillance. Patients receiving postoperative adjuvant chemotherapy underwent tumour marker measurement (carcinoembryonic antigen and colorectal carcinoma antigen) monthly for the first 6 months, then every 3 months until 3 years after surgery and every 6 months thereafter. Patients not receiving postoperative adjuvant chemotherapy underwent tumour marker measurement every 3 months. Chest and abdominal computed tomography was performed every 3 months for the first 3 years, every 6 months for the following 2 years and annually thereafter.

\section{Histological assessment and immunohistochemistry of pulmonary metastasis of PDAC}

In patients who underwent pulmonary resection, the metastatic lesion was differentiated from primary lung cancer by immunohistochemistry, and the final diagnosis was confirmed in resected specimens pathologically using cytokeratin (CK) 7, CK20, thyroid transcription factor-1 (TTF-1), and napsin A. Pulmonary metastasis of PDAC has been reported to be CK7- and CK20-positive, but TTF-1- and napsin A-negative by immunostaining [16-18].

\section{Statistical analysis}

All of the statistical analyses were performed using a standard statistical program (SPSS software for windows, version 26.0; Chicago, IL, USA). Chi-square and MannWhitney $U$ tests were used to analyse patient parameters. OS and recurrence-free survival (RFS) were analysed using the Kaplan-Meier method, and statistical significance was evaluated by the log-rank test. A p-value less than 0.05 was considered statistically significant.

\section{Results}

Baseline characteristics of patients with pulmonary metastasis of PDAC

Of the 417 patients with resected PDACs, 304 (72.9\%) experienced recurrence during the observation period. Only $24(7.9 \%)$ patients had pulmonary metastases as the first recurrence. The median observation period was 44 months (10-153 months). Baseline characteristics of these 24 patients at the time of surgical resection of the primary PDAC are shown in Table 1. Fifteen men and 9 women were included in our study, with a median age of 69 years (55-80 years). Gemcitabine combined with S-1 therapy was administered to two patients as neoadjuvant chemotherapy (8.3\%). For resection of the primary tumour, patients underwent pancreaticoduodenectomy $(\mathrm{n}=17 ; 70.8 \%)$ or distal pancreatectomy $(\mathrm{n}=7 ; 29.2 \%)$. Six patients $(25.0 \%)$ underwent portal vein resection and reconstruction. Five, 1,8 , and 10 patients had TNM stage $1 \mathrm{~A}, 1 \mathrm{~B}, 2 \mathrm{~B}$, and 3 disease, respectively. For adjuvant chemotherapy, patients received gemcitabine $(n=4)$, gemcitabine combined with $S-1(n=3)$ or S-1 $(n=14)$; three patients opted not to receive adjuvant chemotherapy. If recurrence was detected during routine follow-up, the regimen was changed. After the diagnosis of pulmonary metastasis, 6 patients $(25.0 \%)$ underwent pulmonary resection and $18(75.0 \%)$ received chemotherapy or best supportive care. Best supportive care was provided to three patients with poor performance status and one patient with renal dysfunction who could not receive chemotherapy. The median diameter of the largest tumour in the pulmonary metastases was $11.5 \mathrm{~mm}$ (3-32 mm).

A single metastasis was observed in 1 case, $2-5$ metastases in 11 cases, 6-9 metastases in four cases, 10-19 metastases in 3 cases, and more than 20 metastases in five cases. Of the six pneumonectomies, two were biopsied preoperatively to confirm the diagnosis of 
Table 1 Baseline characteristics of patients with metachronous pulmonary metastases from PDAC

\begin{tabular}{|c|c|}
\hline Variables & \\
\hline Age, years, median (range) & $69.0(55.0-80.0)$ \\
\hline \multicolumn{2}{|l|}{ Sex, n (\%) } \\
\hline Male & $15(56.0)$ \\
\hline Female & $9(44.0)$ \\
\hline \multicolumn{2}{|l|}{ ECOG performance status, n (\%) } \\
\hline 0,1 & $21(87.5)$ \\
\hline 2,3 & $3(12.5)$ \\
\hline \multicolumn{2}{|l|}{ Location, n (\%) } \\
\hline Head & $17(70.8)$ \\
\hline Body-tail & $7(29.2)$ \\
\hline CEA (ng/mL), median (range) & $3.9(1.2-51.5)$ \\
\hline CA19-9 (U/mL), median (range) & $128.5(11.0-1185.9)$ \\
\hline \multicolumn{2}{|l|}{ Neoadjuvant chemotherapy, n (\%) } \\
\hline Gemcitabine + S-1 & $2(8.3)$ \\
\hline \multicolumn{2}{|l|}{ Surgical procedure, n (\%) } \\
\hline PD & $17(70.8)$ \\
\hline DP & $7(29.2)$ \\
\hline Portal vein resection, $\mathrm{n}(\%)$ & $6(25.0)$ \\
\hline Primary tumour size (mm), median (range) & $31(6-80)$ \\
\hline \multicolumn{2}{|l|}{ Pathologic T category (primary tumour), n (\%) } \\
\hline $\mathrm{T} 1$ & $6(25.0)$ \\
\hline $\mathrm{T} 2$ & $11(45.8)$ \\
\hline T3 & $7(29.2)$ \\
\hline \multicolumn{2}{|l|}{ Pathologic N category, n (\%) } \\
\hline No & $6(25.0)$ \\
\hline N1 & $8(33.3)$ \\
\hline N2 & $10(41.7)$ \\
\hline \multicolumn{2}{|l|}{ Residual tumour category, n (\%) } \\
\hline RO & $21(87.5)$ \\
\hline R1 & $3(12.5)$ \\
\hline \multicolumn{2}{|l|}{ Tumour differentiation, n (\%) } \\
\hline Well & $9(37.5)$ \\
\hline Moderate & $11(45.8)$ \\
\hline Poor & $4(16.7)$ \\
\hline \multicolumn{2}{|l|}{ Pathologic UICC stage, n (\%) } \\
\hline $1 \mathrm{~A}$ & $5(20.8)$ \\
\hline 1B & $1(4.2)$ \\
\hline $2 \mathrm{~A}$ & 0 \\
\hline $2 \mathrm{~B}$ & $8(33.3)$ \\
\hline 3 & $10(41.7)$ \\
\hline Adjuvant chemotherapy, n (\%) & $21(87.5)$ \\
\hline S-1 & $14(58.3)$ \\
\hline Gemcitabine & $4(16.7)$ \\
\hline Gemcitabine + S-1 & $3(12.5)$ \\
\hline
\end{tabular}

$P D A C$ pancreatic ductal adenocarcinoma, $P D$ pancreatoduodenectomy, $D P$ distal pancreatectomy, ECOG Eastern Cooperative Oncology Group, UICC Union for International Cancer Control, CEA carcinoembryonic antigen, CA19-9 colorectal cancer antigen

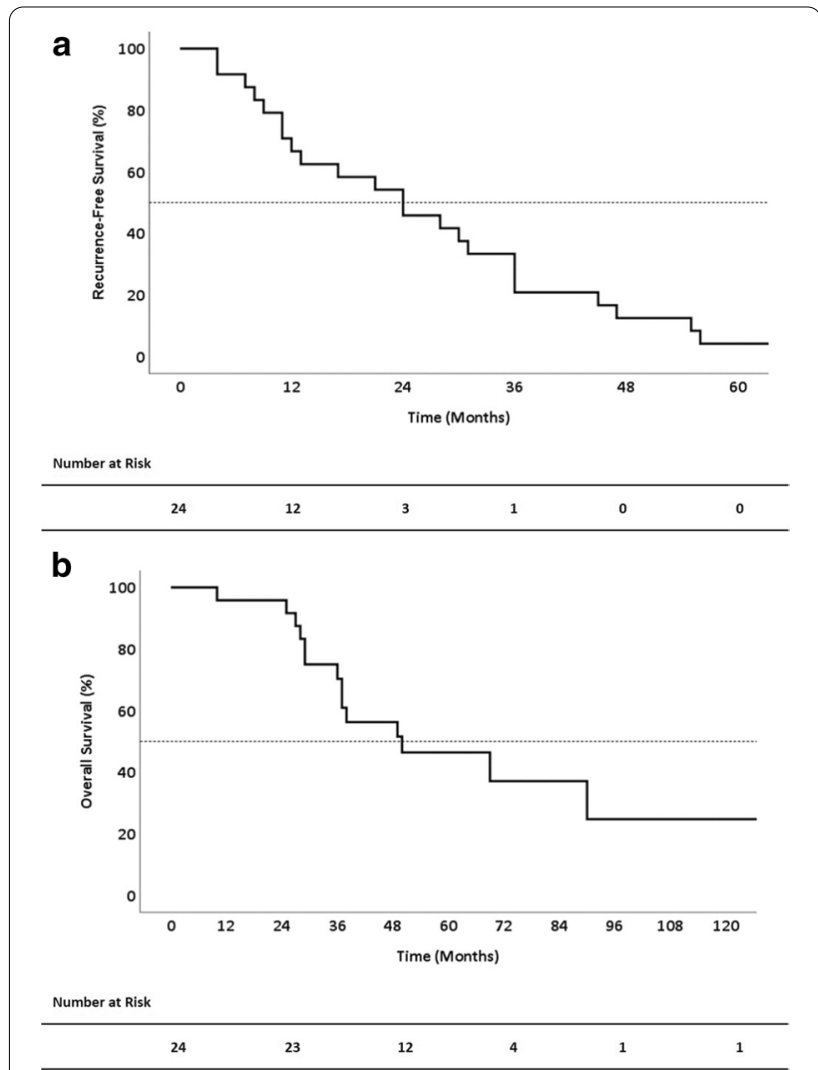

Fig. 1 Survival of patients with pulmonary metastases after resection of primary PDAC. a Recurrence-free survival and $\mathbf{b}$ Overall survival. PDAC pancreatic adenocarcinoma

pulmonary metastases from PDAC. The other four cases were not clearly differentiated from primary lung cancer and were determined only by computed tomography. In our department, the indication for resection of pulmonary metastases is good performance status and localised and curative resection regardless of single or multiple metastases. On the other hand, patients in a poor general condition, patients with early recurrence (within 1 year of surgery), and patients with multiple metastases that were not localised were excluded from surgery.

\section{RFS and OS of all patients}

The median RFS of all 24 patients was 24.0 months (10.8-37.2 months), and the 1-, 3-, and 5-year RFS rates were $66.7 \%, 33.3 \%$ and $4.2 \%$, respectively (Fig. 1a). The median OS was 50.0 months (15.9-84.1 months), and the 1 -, 3-, and 5-year OS rates were $95.8 \%, 70.3 \%$, and $46.4 \%$, respectively (Fig. 1b). 


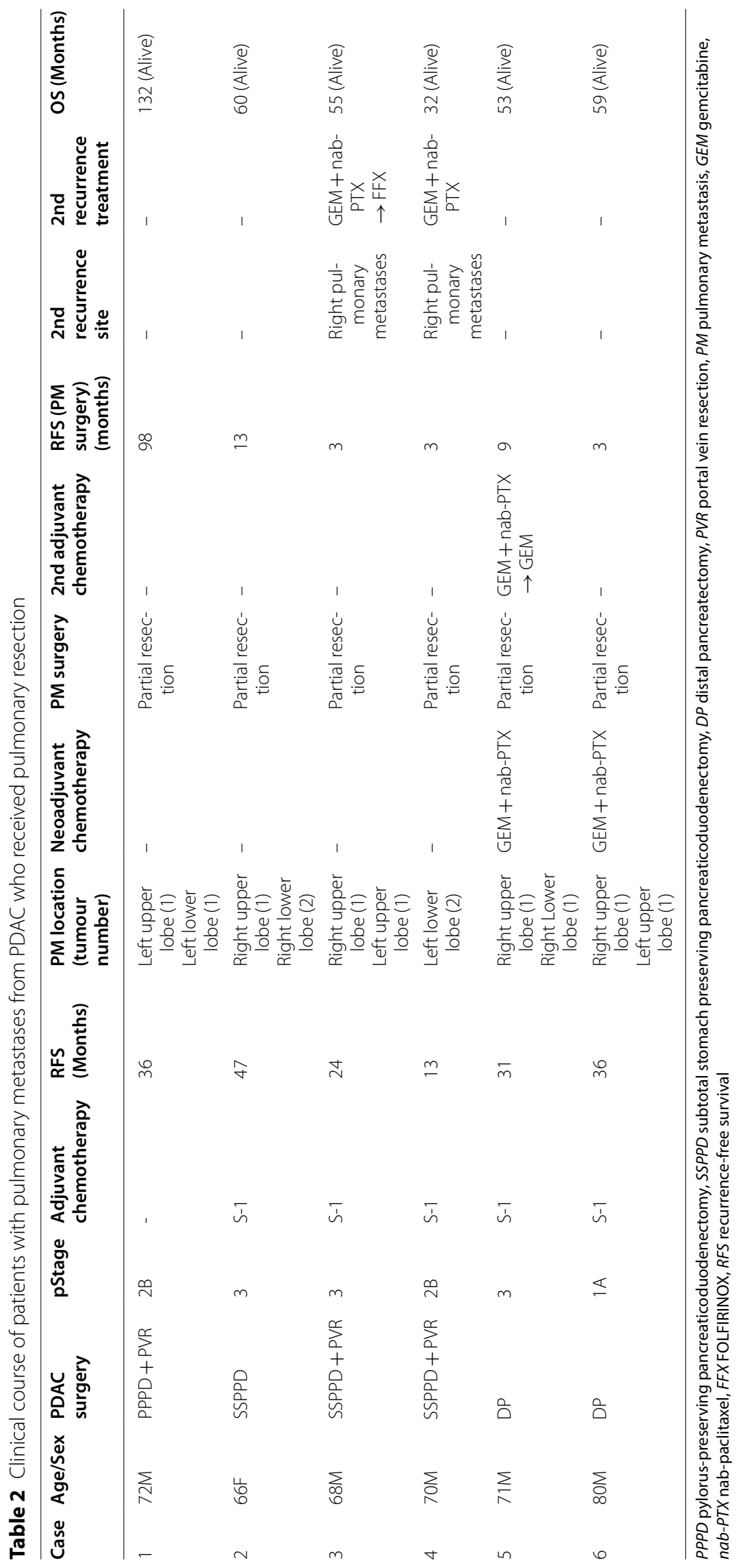


Treatment of patients with pulmonary metastasis of PDAC Table 2 shows the clinical courses of the six patients who underwent pulmonary resection. No lobectomies were performed, and all patients underwent video-assisted thoracoscopic partial pulmonary resection. At the time of the first operation, resection of a single nodule was performed in four patients, whereas two patients underwent resection of two or more lesions. Three patients underwent two or more pulmonary resections. In all cases of resection of pulmonary metastases, the resection margins were negative. The median tumour diameter was $13 \mathrm{~mm}$ (3-31 mm). CK-7, CK20, and TTF-1 assays were performed for all patients. Napsin A and CA19-9 immunostainings were performed in two and three patients, respectively. The metastases were finally diagnosed by two pathologists as consistent with pulmonary metastases of PDAC. In this study, no lung lesions were diagnosed as primary lung cancer.

Table 3 compares the clinicopathological features of patients who did and did not undergo pulmonary resection. Patients who underwent pulmonary resection were significantly more likely to have unilaterally localised disease $(p=0.006)$, but there was no significant difference between the two groups in any other clinicopathological features.

\section{Postoperative complications of pulmonary resection}

Complications included pleural effusion in one patient. No patients had complications of Clavien-Dindo category 3 or higher, and no patients required transfusion. The median hospital stay was 9 days (7-11 days).

\section{Univariate analysis of prognostic factors}

There was no significant difference in prognosis between patients with single and multiple pulmonary metastases $(\mathrm{p}=0.523)$. The OS was 36 months $(95 \%$ CI 27.2-44.8 months) and 90.0 months (95\% CI 49.9130.1 months), respectively, for patients who developed pulmonary metastases $\leq 24$ months and $\geq 24$ months after resection of the primary tumour $(\mathrm{p}<0.001)$ (Fig. 2a). The OS was 90 months (95\% CI 16.9-163.1) and 37 months (95\% CI 23.6-50.4), respectively, for patients with primary tumours $\leq 31 \mathrm{~mm}$ and those with tumours $>31 \mathrm{~mm}(\mathrm{p}=0.018)$ (Fig. $2 \mathrm{~b})$. There was no difference in prognosis according to the presence or absence of lymph node metastasis $(p=0.213)$. However, when comparing patients with $\mathrm{N} 2$ disease with four or more regional lymph node metastases to patients with N0 and N1 disease, the OS was 37 months (95\% CI 25.948.1) and 90 months (95\% CI 15.0-165.0), respectively $(\mathrm{p}=0.046)$ (Fig. 2c). The median RFS was 31 months (95\% CI 21.4-40.6) and 17 months (95\% CI 0.0-35.7), respectively, for patients who underwent pulmonary resection and those who did not. Although there was no significant difference in disease-free survival (DFS) in the small number of cases studied $(\mathrm{p}=0.643)$, early recurrence within 1 year occurred more frequently in the no pulmonary resection group (eight cases). This group also

Table 3 Baseline characteristics of the patients who did or did not receive pulmonary resection

\begin{tabular}{|c|c|c|c|}
\hline Variables & Pulmonary resection $(n=6)$ & $\begin{array}{l}\text { No pulmonary resection } \\
(\mathrm{n}=18)\end{array}$ & p-value \\
\hline Age, years, median (range) & $70.5(66.0-80.0)$ & $70(55.0-80.0)$ & 0.251 \\
\hline Sex (Male/Female) & $5 / 1$ & $10 / 8$ & 0.238 \\
\hline ECOG Performance status $(0,1 / 2,3)$ & $6 / 0$ & $21 / 3$ & 0.403 \\
\hline CEA (ng/mL), median (range) & $4.9(1.5-51.5)$ & $3.7(1.2-13.0)$ & 0.251 \\
\hline CA19-9 (U/mL), median (range) & $249.3(18.0-955.1)$ & $90.1(11-1185.9)$ & 0.224 \\
\hline Primary tumour location (Head/Body-tail) & $4 / 2$ & $11 / 7$ & 0.603 \\
\hline Primary tumour size, median (range) & $32.5(20.0-80)$ & $31(6.0-50.0)$ & $>0.99$ \\
\hline Pathologic $T$ category of primary tumour $(\mathrm{T} 1,2 / \mathrm{T} 3)$ & $4 / 2$ & $13 / 5$ & 0.586 \\
\hline Pathologic $\mathrm{N}$ category of primary tumour $(\mathrm{N} 0,1 / \mathrm{N} 2)$ & $4 / 2$ & $11 / 7$ & 0.603 \\
\hline Pathologic UICC Stage $(1,2 / 3)$ & $3 / 3$ & $11 / 7$ & 0.494 \\
\hline Residual tumour category (R0/R1) & $6 / 0$ & $15 / 3$ & 0.403 \\
\hline Tumour differentiation (Well/Moderate, poor) & $2 / 4$ & $7 / 11$ & 0.603 \\
\hline Completion of adjuvant chemotherapy, n (\%) & $3 / 3$ & $5 / 13$ & 0.302 \\
\hline $\begin{array}{l}\text { Tumour diameter of pulmonary metastases }(\mathrm{mm}) \text {, median } \\
\text { (range) }\end{array}$ & $13.0(3.0-31.0)$ & $11.5(4.0-32.0)$ & 0.923 \\
\hline Number of PM (solitary/multiple) & $0 / 6$ & $1 / 17$ & 0.750 \\
\hline Site of PM (unilateral/bilateral) & $4 / 2$ & $1 / 17$ & 0.006 \\
\hline
\end{tabular}

CEA carcinoembryonic antigen, CA19-9 colorectal cancer antigen, UICC Union for International Cancer Control, PM pulmonary metastases, ECOG Eastern Cooperative Oncology Group 


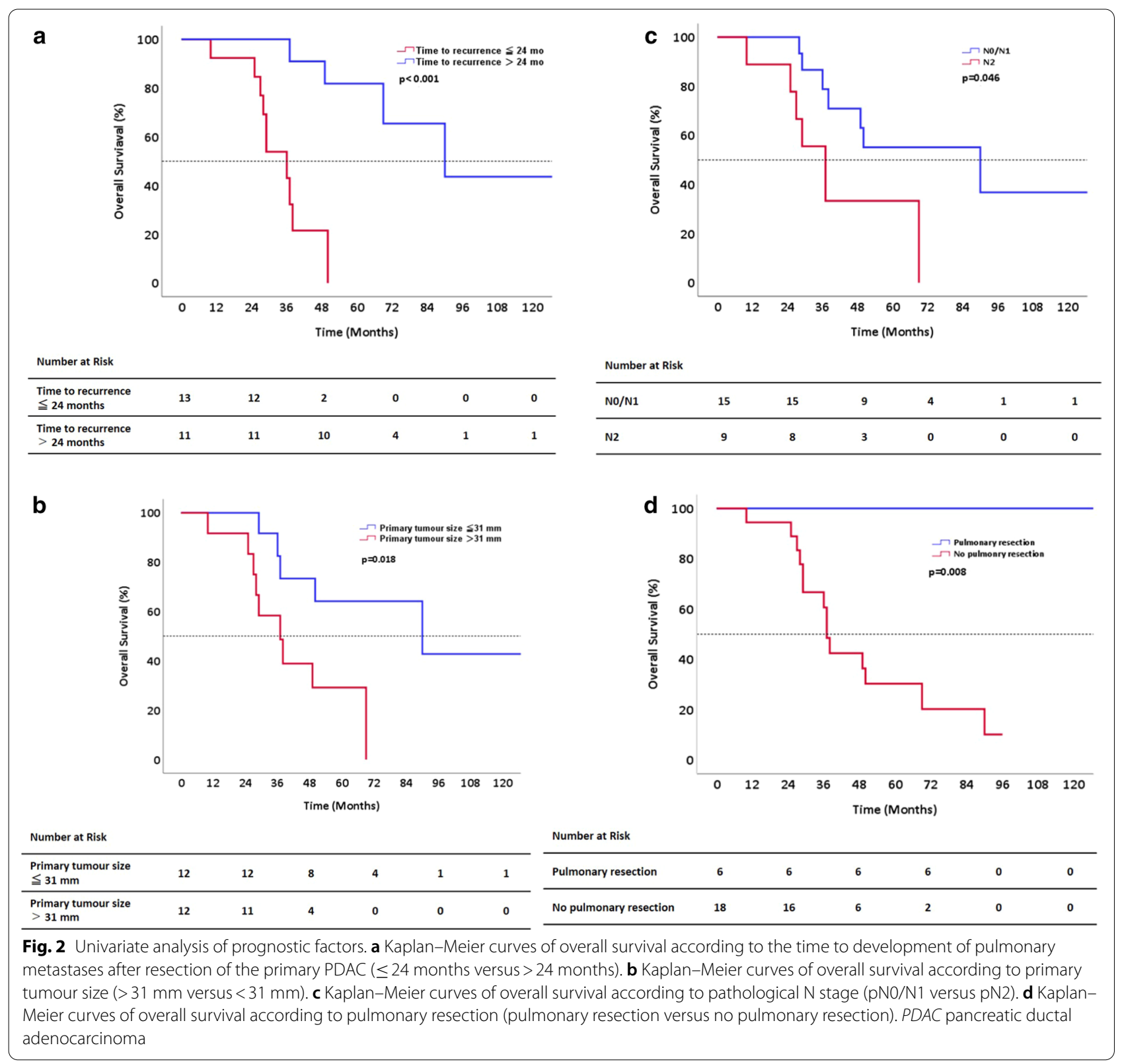

had a shorter DFS. Conversely, all patients with resected pulmonary metastases survived, whereas the OS of patients who did not receive pulmonary resection was 37.0 months (95\% CI 34.4-39.6), indicating a significantly better prognosis of patients who underwent pulmonary metastasectomy $(\mathrm{p}=0.008)$ (Fig. 2d).

\section{Discussion}

PDAC can be cured only by multidisciplinary treatment with surgery and postoperative adjuvant chemotherapy. However, the recurrence rate is high, with $80 \%$ of patients reporting a relapse within 2 years [5]. Additionally, PDAC often metastasises to multiple organs by hematogenous, lymphogenic, and perineural routes or by direct invasion $[19,20]$.

According to a meta-analysis including 83 studies, the liver is the most common site of metastasis of PDAC, accounting for $26.5 \%$ of recurrences, followed by local recurrence $(20.8 \%)$, peritoneal dissemination (13.5\%), and lung metastasis (11.4\%) [21]. The mechanism underlying pulmonary metastasis as the first recurrence is not well understood, but it has been suggested that this occurs through lymphatic entry into 
the macrocirculation from lymph node metastasis or hematogenous metastasis via the collateral blood circulation around the spleen due to congestion of the splenic vein [22].

The time to recurrence has been reported to be longer in patients with pulmonary metastasis as the first recurrence than in patients with other sites of recurrence [9$11,14,15,21,22]$. Zheng et al. noted that these patients are more likely to have a lower pathological $\mathrm{T}$ category and less vascular invasion than patients with metastases at other sites [22]. In our study, the median RFS and OS of patients with pulmonary metastases were 24 months and 50 months, respectively, similar to those in previous reports $[7,14,22]$. On the contrary, most of the cases in our study were T2 or larger with relatively large tumour diameters.

Resection of recurrent PDAC lesions is usually not indicated, and systemic chemotherapy is administered instead. However, in our study, the prognosis of patients with resected pulmonary metastases was significantly better than that of patients who did not undergo resection. Arnautakis et al. reported that nine patients who underwent pulmonary resection had a longer OS than 22 patients who did not (51 months versus 23 months, $\mathrm{p}=0.04)$. They also demonstrated that pulmonary metastasectomy could be performed safely without major postoperative complications [7]. In our study, pulmonary resection was performed safely, and there were no complications requiring therapeutic intervention. Although all of our pneumonectomies were video-assisted thoracoscopic partial resections, we believe that pneumonectomy can be safely performed even after PDAC resection in patients who are in a good general condition. Thomford et al. advocated the following indications for the resection of pulmonary metastases: (1) tolerability of surgery, (2) controlled primary lesion, (3) no metastases in other organs, and (4) radiographic evidence of unilateral pulmonary metastasis [23].

Patients with poorly differentiated PDAC at the time of the initial surgery have a poor prognosis [24], and patients with pulmonary metastatic lesions less than $16 \mathrm{~mm}$ in size and stage 1 primary tumours have a better prognosis [25]. By contrast, Kruger et al. considered that patients with fewer than 10 localised metastases on one side of the lung had a good prognosis and that the size of each metastasis was not an important factor in planning pulmonary resection [10]. In our study, the prognosis was worse in patients with larger tumours and more than four metastatic lymph nodes in the primary PDAC. However, there was only one case of single metastasis. Therefore, it was difficult to determine if single or multiple metastases affected prognosis in this study. Thomas et al. reported a significantly better prognosis in patients with recurrence $\geq 21$ months after PDAC resection than in those with recurrence $\leq 21$ months after PDAC resection (92.3 months versus 31.3 months, $\mathrm{p}=0.033$ ). In addition, Ilmer et al. reported that the prognosis of patients with recurrence $\geq 17$ months after PDAC resection was significantly more favourable than that of patients with recurrence $\leq 17$ months after PDAC among patients undergoing pneumonectomy (32.2 months versus 14.8 months, $\mathrm{p}=0.025)[24,26]$. Our study also showed a significantly better prognosis for patients who relapsed $\geq 24$ months after PDAC resection. When examining patients who underwent pneumonectomy, two patients with recurrence $\leq 24$ months after PDAC showed early recurrence after pneumonectomy. Therefore, the time to recurrence is an important factor when considering the indication for pneumonectomy.

Patients whose pulmonary metastases have been wellcontrolled by chemotherapy are also good candidates for surgery, but there is insufficient evidence on whether and when to perform pneumonectomy after chemotherapy. Two patients underwent resection after neoadjuvant chemotherapy for pulmonary metastases: one patient was treated with gemcitabine plus nab-paclitaxel for more than one year and was found to have no disease progression, and the other, who was older, was treated with gemcitabine plus nab-paclitaxel for one month before undergoing surgery at his request. Nakajima et al. also mentioned that adjuvant chemotherapy after curative resection of pulmonary metastasis from PDAC was controversial [25]. Therefore, only one of our patients received postoperative adjuvant chemotherapy after pneumonectomy, and the other five patients were only followed up. Despite the long duration of this study, there were no major changes in the techniques used or extent of lymph node dissection. Eleven patients received gemcitabine plus nab-paclitaxel and seven patients received other regimens. However, no bias was observed in the regimens between the two groups $(\mathrm{p}=0.103)$, and thus, this did not affect the results of the study.

There are some limitations to this study. First, this is a retrospective study. Second, this study is based on a small number of cases and there may be selection bias. In addition, the no pulmonary resection group included cases of best supportive care. Due to the limited number of patients, we were unable to identify prognostic factors in patients with pulmonary metastases from PDAC using multivariate analysis. Further multicenter prospective studies are needed to determine the factors associated with prognosis in patients with pulmonary metastases from PDAC who undergo metastasectomy. 


\section{Conclusions}

Despite the limitations mentioned in the Discussion, our study showed that pulmonary resection may further improve prognosis in selected patients with pulmonary metastases from PDAC. In particular, patients with a longer time to recurrence for whom radial resections are suitable are considered to be good candidates for pulmonary resection. A multi-institutional prospective study is needed to clarify the indications for pulmonary resection.

\section{Abbreviations}

PDAC: Pancreatic ductal adenocarcinoma; CK: Cytokeratin; TTF-1: Thyroid transcription factor-1; RFS: Relapse-free survival; OS: Overall survival; DFS: Diseasefree survival; TNM: Tumour-node-metastasis; Cl: Confidence interval.

\section{Acknowledgements}

We thank the Department of Respiratory Surgery for performing pulmonary resection.

\section{Authors' contributions}

TM analysed and interpreted the patient data and was a major contributor in writing the manuscript. TN, YM, AN, SY, OS reviewed and edited the manuscript. All authors read and approved the final manuscript.

\section{Funding}

Not applicable.

\section{Availability of data and materials}

The datasets generated and/or analysed during the current study are available from the corresponding author on reasonable request.

\section{Declarations}

\section{Ethics approval and consent to participate}

All methods were performed in accordance with the Declaration of Helsinki. The present study was approved by the Institutional Ethical Board of Tokai University Hospital. The requirement for informed consent was waived by the Institutional Ethical Board of Tokai University Hospital due to the retrospective nature of the study.

\section{Consent for publication}

Not applicable.

\section{Competing interests}

The authors declare that they have no competing interests.

Received: 10 February 2021 Accepted: 28 April 2021

Published online: 05 May 2021

\section{References}

1. Katz MH, Wang H, Fleming JB, Sun CC, Hwang RF, Wolff RA, et al. Longterm survival after multidisciplinary management of resected pancreatic adenocarcinoma. Ann Surg Oncol. 2009;16:836-47.

2. Vincent A, Herman J, Schulick R, Hruban RH, Goggins M. Pancreatic cancer. Lancet. 2011;378:607-20.

3. Von Hoff DD, Ervin T, Arena FP, Chiorean EG, Infante J, Moore M, et al. Increased survival in pancreatic cancer with nab-paclitaxel plus gemcitabine. N Engl J Med. 2013;369:1691-703.

4. Conroy T, Desseigne F, Ychou M, Bouché O, Guimbaud R, Bécouarn Y, et al. FOLFIRINOX versus gemcitabine for metastatic pancreatic cancer. N Engl J Med. 2011;364:1817-25.

5. Groot VP, Rezaee N, Wu W, Cameron JL, Fishman EK, Hruban RH, et al. Patterns, timing and predictors of recurrence following pancreatectomy for pancreatic ductal adenocarcinoma. Ann Surg. 2018;267:936-45.
6. Wangjam T, Zhang Z, Zhou XC, Lyer L, Faisal F, Soares KC, et al. Resected pancreatic ductal adenocarcinomas with recurrence limited in lung have a significantly better prognosis than those with other recurrence patterns. Oncotarget. 2015;6:36903-10.

7. Arnaoutakis GJ, Rangachari D, Laheru DA, lacobuzio-Donahue CA, Hruban $\mathrm{RH}$, Herman JM, et al. Pulmonary resection for isolated pancreatic adenocarcinoma metastasis: an analysis of outcomes and survival. J Gastrointest Surg. 2011;15:1611-7.

8. Yamashita K, Miyamoto A, Hama N, Asaoka T, Maeda S, Omiya H, et al. Survival impact of pulmonary metastasis as recurrence of pancreatic ductal adenocarcinoma. Dig Surg. 2015;32:464-71.

9. Deeb A, Haque SU, Olowokure O. Pulmonary metastases in pancreatic cancer, is there a survival influence? J Gastrointest Oncol. 2015;6:E48-51.

10. Kruger S, Haas M, Burger PJ, Ormanns S, Modest DP, Westphalen CB, et al. Isolated pulmonary metastases define a favorable subgroup in metastatic pancreatic cancer. Pancreatology. 2016;16:593-8.

11. Decoster C, Gilabert M, Autret A, Turrini O, Oziel-Taieb S, Poizat F, et al. Heterogeneity of metastatic pancreatic adenocarcinoma: lung metastasis show better prognosis than liver metastasis-a case control study. Oncotarget. 2016;7:45649-55.

12. Brieau B, Barret M, Rouquette A, Dréanic J, Brezault C, Regnard JF, et al. Resection of late pulmonary metastases from pancreatic adenocarcinoma. Is surgery an option? Cancer Investig. 2015;33:522-5.

13. Lu F, Poruk KE, Weiss MJ. Surgery for oligometastasis of pancreatic cancer. Chin J Cancer Res. 2015;27:358-67.

14. Lovecek M, Skalicky P, Chudacek J, Szkorupa M, Svebisova H, Lemstrova R, et al. Different clinical presentations of metachronous pulmonary metastases after resection of pancreatic ductal adenocarcinoma: retrospective study and review of the literature. World J Gastroenterol. 2017;23:6420-8.

15. Kurahara H, Maemura K, Mataki Y, Tanoue K, lino S, Kawasaki Y, et al. Lung recurrence and its therapeutic strategy in patients with pancreatic cancer. Pancreatology. 2020;20:89-94.

16. Park SY, Kim BH, Kim JH, Lee S, Kang GH. Panels of immunohistochemical markers help determine primary sites of metastatic adenocarcinoma. Arch Pathol Lab Med. 2007;131:1561-7.

17. Nakamura N, Miyagi E, Murata S, Kawaoi A, Katoh R. Expression of thyroid transcription factor-1 in normal and neoplastic lung tissues. Mod Pathol. 2002;15:1058-67.

18. Krasinskas AM, Chiosea SI, Pal T, Dacic S. KRAS mutational analysis and immunohistochemical studies can help and distinguish pancreatic metastases from primary lung adenocarcinomas. Mod Pathol. 2014;27:262-70.

19. Kamisawa T, Isawa T, Koike M, Tsuruta K, Okamoto A. Hematogenous metastases of pancreatic ductal carcinoma. Pancreas. 1995;11:345-9.

20. Yachida S, lacobuzio-Donahue CA. The pathology and genetics of metastatic pancreatic cancer. Arch Pathol Lab Med. 2009;133:413-22.

21. Tanaka M, Mihaljevic AL, Probst P, Heckler M, Klaiber U, Heger U, et al. Meta-analysis of recurrence pattern after resection for pancreatic cancer. Br J Surg. 2019;106:1590-601.

22. Zheng B, Ohuchida K, Yan Z, Okumura T, Ohtsuka T, Nakamura M. Primary recurrence in the lung is related to favorable prognosis in patients with pancreatic cancer and postoperative recurrence. World J Surg. 2017;41:2858-66.

23. Thomford NR, Woolner LB, Clagett OT. The surgical treatment of metastatic tumours in the lungs. J Thorac Cardiovasc Surg. 1965;49:357-63.

24. Ilmer M, Schiergens TS, Renz BW, Schneider C, Sargut M, Waligora R, et al. Oligometastatic pulmonary metastasis in pancreatic cancer patients: safety and outcome of resection. Surg Oncol. 2019;31:16-21.

25. Nakajima M, Ueno T, Suzuki N, Matsui H, Shindo Y, Sakamoto K, et al. Novel indications for surgical resection of metachronous lung metastases from pancreatic cancer after curative resection. J Clin Gastroenterol. 2017:51:e34-38

26. Thomas RM, Truty MJ, Nogueras-Gonzalez GM, Fleming JB, Vauthey JN, Pisters PW, et al. Selective reoperation for locally recurrent or metastatic pancreatic ductal adenocarcinoma following primary pancreatic resection. J Gastrointest Surg. 2012;16:1696-704.

\section{Publisher's Note}

Springer Nature remains neutral with regard to jurisdictional claims in published maps and institutional affiliations. 